\title{
Echocardiographic Assessment at 1-Year Follow-Up Post-Percutaneous Pulmonary Valve Implantation at the Prince Sultan Cardiac Center: A Single-Center Experience
}

\author{
Al Asmari Own*, Atiyah Merna, Al Essa Mohammed, Almohaizy \\ Omar, Al Sahari Atif, Mohsin Shazia, Al Khalaf Khalaf and Al Najashi \\ Khalid \\ Department of Pediatric Cardiology, Prince Sultan Cardiac Center, Riyadh, Kingdom \\ of Saudi Arabia \\ *Corresponding Author: Al Asmari Own, Department of Pediatric Cardiology, \\ Prince Sultan Cardiac Center, Riyadh, Kingdom of Saudi Arabia.
}

DOI: $10.31080 /$ ASMS.2020.04.0690

\begin{abstract}
Background: Congenital heart defects affect roughly 1 in 150 people and are often associated with right ventricular outflow tract (RVOT) dysfunction. The aim of the study was to evaluate the echocardiographic changes within the right ventricle following percutaneous pulmonary valve implantation (PPVI).

Methods: The echocardiographic data of 57 patients who had pulmonary valve stenosis and/or regurgitation and had undergone PPVI treatment and completed at least 1 year of regular follow-up at the Prince Sultan Cardiac Center from January 2012 to December 2015 were analyzed at the baseline level (before PPVI) and at 6 and 12 months following the PPVI treatment. Pulmonary insufficiency was graded from 0 to 4 based on severity: 0 , none; 1 , trivial; 2 , mild; 3 , moderate; 4 , severe/free).

Results: There was a significant reduction in the RVOT gradient. At baseline, 95\% of the subjects demonstrated pulmonary insufficiency (grades 2-4); at 12 months, 28\% had decreased pulmonary insufficiency and $72 \%$ had limited to no pulmonary regurgitation. Other parameters, including right ventricular volumes, fractional area change, and the tricuspid regurgitant jet width/body surface area, did not demonstrate any significant change. Early follow-up echocardiographic data demonstrated persistent improvement in the RVOT gradient and pulmonary regurgitation level over the 1-year follow-up.

Conclusion: PPVI was seen to ameliorate RVOT dysfunction in most subjects.
\end{abstract}

Keywords: Congenital Heart Defects; Right Ventricular Outflow Tract; Percutaneous Valve Implantation; Echocardiography

\section{Introduction}

The prevalence of congenital heart defects has been estimated as 1 in 150 individuals in the adult population [1]. It has been further identified that the incidence of right ventricular outflow tract (RVOT) defect in newborns is also increasing, which results in congenital heart defects such as truncus arteriosus, tetralogy of Fallot, and pulmonary atresia. Hence, these defects need to be addressed via reconstructive surgery, incorporating the use of a patch, valved conduits, or bioprosthetic valves. Studies have noted that reconstructive surgeries that use conduits or bioprosthetic valves are commonly used to treat RVOT disorder in children with complex heart disorders [2-4]. However, various complications may arise from such techniques. 
Echocardiographic Assessment at 1-Year Follow-Up Post-Percutaneous Pulmonary Valve Implantation at the Prince Sultan Cardiac Center: A Single-Center Experience

The incidence of pulmonary regurgitation $(\mathrm{PR})$ in patients who underwent post-RVOT reconstruction using transannular patches is also high [1]. Moreover, the biological material utilized in bioprosthesis and valved conduits is prone to degeneration, leading to progressive RVOT dysfunction including PR and pulmonary stenosis [5-7]; thus, patients have to undergo repeated surgical interventions. Further studies discussed the limited longevity of RVOT reconstruction [8-10] and highlighted PR as a risk factor for long-term morbidity and mortality [11-13], along with the primary indication for reoperation $[14,15]$. Although surgical outflow tract revision is associated with a low morbidity and mortality [11-15], such replacements have a limited life span and require additional open-heart procedures. Therefore, a suitable alternative that would adequately address obstructive or regurgitant lesions in RVOT reconstruction surgery is essential. Bonhoeffer., et al. [16] introduced percutaneous pulmonary valve implantation (PPVI) as a non-surgical treatment for RVOT dysfunction. The use of two types of valves was noted, namely, Melody Medtronic and Edwards SAPIEN transcatheter heart valves [1]. PPVI has been used in more than 10,000 reconstruction procedures conducted in 200 centers in 35 countries [1]. One of the largest reported experience was noted in 155 patients who had undergone PPVI at a median age of 21 years [17], which showed that the PPVI was technically successful with a low complication rate, resulting in marked improvement of the right ventricular (RV) hemodynamic status [17]. Therefore, this study was conducted an early-term follow-up of certain echocardiographic parameters in older children, adolescents, and the adult population post-PPVI treatment.

\section{Methods}

Following the initiation of PPVI treatment at the Prince Sultan Cardiac Center in Riyadh, Saudi Arabia, a well-organized program on pre-implantation workup and post-implantation follow-up via echocardiography at regular time intervals was established. This study included 57 of 92 patients who underwent PPVI treatment at the center from January 2012 to December 2015 and completed at least 1-year follow-up. Regular echocardiographic assessment was carried out for these patients at specific time intervals. The first assessment was conducted within 6 months before the implantation process. The next assessments were carried out 6 and 12 months following the implantation process. Two-dimensional (2D) Doppler quantification echocardiographic data were obtained from the system.

Both the RV functional status and the degree of dilation were assessed in all the patients, using a Philips IE-33 2D transthoracic echocardiographic system. The right ventricular end-diastolic di- mensions were obtained via 2D tracing on apical four-chamber view. The study additionally obtained the RV end-diastolic area indexed to the body surface area (RVEDA/BSA) and the RV endsystolic area. RV function was assessed by calculating the RV fractional area change (FAC). An estimate of tricuspid regurgitant (TR) severity was determined from the TR jet width indexed to the body surface area (BSA). The RVOT maximum peak instantaneous gradient was calculated from the velocity flow (CW Doppler interrogation) and the degree of PR was determined qualitatively by the color-flow mapping (CFM) Doppler.

PR was categorized as 0 , none; 1 , trivial (the regurgitant color jet width is less than one third of the pulmonary valve annulus diameter); 2 , mild (the regurgitant color jet width is greater than one third but less than half of the pulmonary valve annulus diameter; 3 , moderate (the regurgitant color jet width is greater than half but less than the pulmonary valve annulus diameter; 4 , severe (the regurgitant color jet width is equal to the pulmonary valve annulus diameter with flow reversal in the branch of the pulmonary arteries). Regurgitation grades 2-4 were considered significant. Data are described as mean \pm standard deviation (SD) or median with minimum and maximum values and percentages, as appropriate.

\section{Results}

This study was conducted on 39 male (68\%) and 18 female $(32 \%)$ subjects, with mean age of $18.8 \pm 7.9$ years (range, $8-45$ years) and mean BSA of $1.5 \mathrm{~m}^{2}$ (range, $1-2.2 \mathrm{~m}^{2}$ ). The results of the baseline echocardiography performed before implantation was compared with those obtained at 6 and 12 months post-implantation. This study noted a significant reduction in the RV-to-pulmonary artery gradient (Table 1 ) from $37 \pm 22.2 \mathrm{~mm} \mathrm{Hg}$ at baseline to $18.2 \pm 6.8 \mathrm{~mm} \mathrm{Hg}$ at the 6-month follow-up and to $18 \pm 6.25 \mathrm{~mm} \mathrm{Hg}$ at the 12-month follow-up (Figure $1 ; \mathrm{p}<0.001$ ).

\begin{tabular}{|l|c|c|c|c|}
\hline & $\begin{array}{c}\text { RVOT }^{\mathbf{a}} \\
\mathbf{P G}^{\mathbf{b}} \mathbf{>} \mathbf{2 5} \\
\mathbf{m m ~ H g}^{\mathbf{m}}\end{array}$ & $\begin{array}{c}\text { RVOT PG } \\
\mathbf{2 5}-\mathbf{5 0} \mathbf{~ m m} \\
\mathbf{H g}\end{array}$ & $\begin{array}{c}\text { RVOT PG } \\
<\mathbf{5 0} \mathbf{~ m m} \\
\mathbf{H g}\end{array}$ & RVOT PG \\
\hline Pre-PPVI $^{\mathrm{c}}$ & $10(17.6)$ & $24(42)$ & $23(40.4)$ & $37.25 \pm 22.2$ \\
\hline 6 months & $50(87.7)$ & $7(12.3)$ & 0 & $18.2 \pm 6.8$ \\
\hline 12 months & $48(84.2)$ & $9(15.8)$ & 0 & $18.64 \pm 6.25$ \\
\hline
\end{tabular}

Table 1: Right ventricular outflow tract peak pressure gradient pre- and post-percutaneous pulmonary valve implantation.

Values are presented as $\mathrm{n}(\%)$ or mean \pm standard deviation.

RVOTa: Right Ventricular Outflow Tract; PG ${ }^{\mathrm{b}}$ : Pressure Gradient; PPVIc: Percutaneous Pulmonary Valve Implantation. 


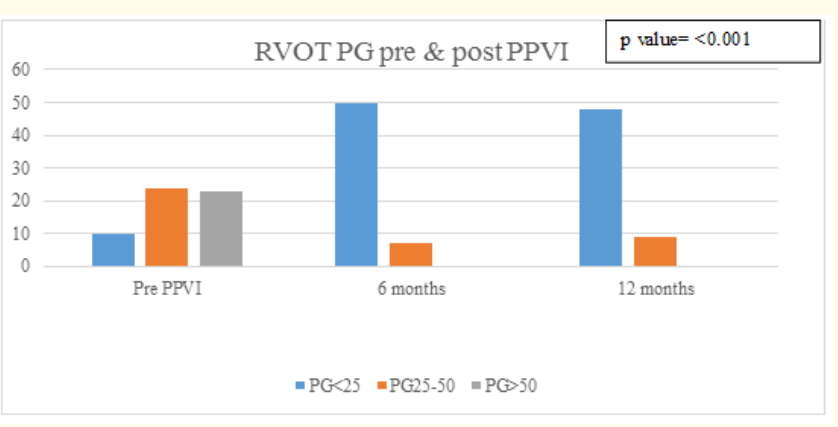

Figure 1: Right ventricular outflow tract pressure gradient (PG) pre- and post-percutaneous valve implantation (PPVI).

There was a significant improvement in the PR level of the investigated subjects following the implantation process (Table 2 and figure 2). At the baseline level, 95\% of the patients $(n=54)$ demonstrated a pulmonary insufficiency grade between 2 and 4 , where $42(82.5 \%)$ patients had moderate to severe PR (grade $\geq 3$ ). However, at the 12-month follow-up after the implantation process, 29 (50.8\%) subjects had no detectable PR ( $\mathrm{p}<0.001), 11$ (19\%) subjects demonstrated trivial PR, and only 2 (3.5\%) subjects exhibited moderate PR (grade 3). None of the subjects developed severe PR (grade 4).

\begin{tabular}{|l|c|c|c|c|c|}
\hline & Grade 0 & Grade 1 & Grade 2 & Grade 3 & Grade 4 \\
\hline $\begin{array}{l}\text { Pre- } \\
\text { PPVI }^{\mathrm{a}}\end{array}$ & $3(5)$ & 0 & $6(10.5)$ & $18(31.5)$ & $30(53)$ \\
\hline $\begin{array}{l}6 \\
\text { months }\end{array}$ & $\begin{array}{c}33 \\
(57.9)\end{array}$ & $9(15.8)$ & $\begin{array}{c}13 \\
(22.8)\end{array}$ & $2(3.5)$ & 0 \\
\hline $\begin{array}{l}12 \\
\text { months }\end{array}$ & $\begin{array}{c}29 \\
(50.9)\end{array}$ & $11(19.3)$ & $\begin{array}{c}15 \\
(26.3)\end{array}$ & $2(3.5)$ & 0 \\
\hline
\end{tabular}

Table 2: Pulmonary regurgitation severity pre- and post-percutaneous pulmonary valve implantation.

Values are presented as n (\%).

PPVIa: Percutaneous Pulmonary Valve Implantation.

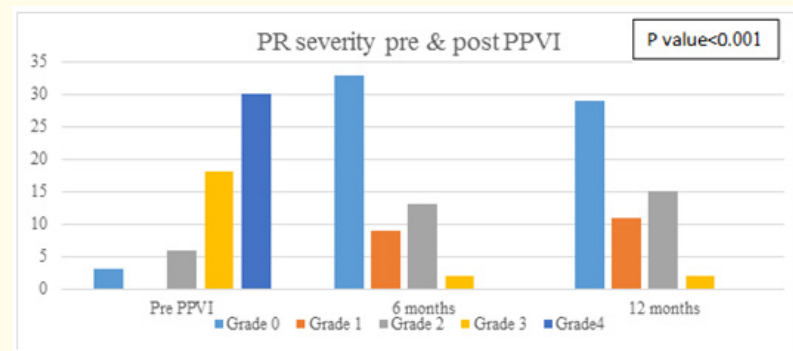

Figure 2: Severity of pulmonary regurgitation severity pre- and post-percutaneous valve implantation (PPVI).
There were no significant changes in tricuspid valve severity (expressed as TR jet width/BSA), RV end-diastolic dimensions (expressed as RVEDA/BSA), and RV function (estimated by FAC) (Table 3 and Figure 3).

\begin{tabular}{|c|c|c|c|}
\hline & TR $^{a}$ jet width/BSA & RVEDA $^{\mathrm{c}} / \mathrm{BSA}$ & FAC $^{\mathrm{d}}$ \\
\hline Pre-PPVI ${ }^{\mathrm{e}}$ & $0.445 \pm 0.24$ & $15.95 \pm 4.8$ & $36.75 \pm 8.2$ \\
\hline 6 months & $0.48 \pm 0.22$ & $13.38 \pm 3.36$ & $36.8 \pm 7.9$ \\
\hline 12 months & $0.5 \pm 0.19$ & $15.6 \pm 14.7$ & $38.5 \pm 7.9$ \\
\hline
\end{tabular}

Table 3: Tricuspid regurgitant, right ventricular end-diastolic area/body surface area, and fractional area change pre- and postpercutaneous pulmonary valve implantation.

Values are presented as mean \pm standard deviation.

TR $^{\mathrm{a}}$ : Tricuspid Regurgitant Jet Width; BSA ${ }^{\mathrm{b}}$ : Body Surface Area; RVEDA': Right Ventricular RV end-diastolic Area; FAC ${ }^{\mathrm{d}}$ : Fractional Area Change; PPVI ${ }^{\mathrm{e}}$ : Percutaneous Pulmonary Valve Implantation.

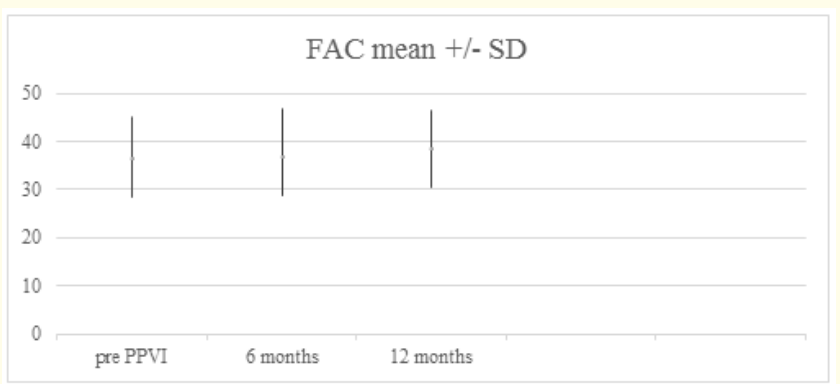

Figure 3: Fractional area change (mean \pm standard deviation) preand post-percutaneous valve implantation (PPVI).

\section{Discussion}

This study has reported the results of the early-term (1-year) echocardiographic follow-up of 57 children, adolescents, and adults who had undergone PPVI treatment for RVOT dysfunction. Therefore, this study has been conducted on subjects belonging to a heterogeneous age set. Moreover, PPVI was demonstrated to be an effective and safe treatment for the amelioration of RVOT dysfunction. This non-surgical approach to treat RVOT dysfunctions that arise as a result of congenital heart defects is seen to be a suitable alternative to current surgical reconstructive modalities that incorporate the use of transannular patches, bioprosthetic valves, and valved conduits.

The use of PPVI has led to a significant relief of RV outflow obstruction and the near-elimination of regurgitation. The longevity of the hemodynamic improvements is a critical aspect in the determination of the impact of this therapy in the clinical course. Therefore, an echocardiographic follow-up was conducted, which 
showed that there was a sustained reduction in the RVOT gradients and PR, thereby eliminating the risk of pressure and volume overload. However, tricuspid valve severity, indexed RVEDA/BSA, and RV function (FAC) did not demonstrate a significant level of change, and only minimal improvement was noted in these parameters. Therefore, longer-term follow-up studies should be conducted to investigate these parameters further. The sustained elimination of progression requires follow-up studies that explore the longerterm effect on these parameters.

This study has some limitations. Although this study has reported the immediate benefits of PPVI through the conduction of an early term follow-up, longer-term follow-up studies can be conducted to determine the longevity of the percutaneous values as compared with surgical interventions. Additionally, this study incorporated subjects that were heterogeneous in terms of their obtained diagnosis and RVOT morphologies, which meant that the subjects were heterogeneous in terms of their diagnosis and RVOT morphology and thus were not categorized. Moreover, the subjects chosen belonged to a rather heterogeneous age set, with many different age groups. Therefore, studies need to be conducted on a larger sample pool to completely ascertain the effectiveness of PPVI treatment in the amelioration of RVOT dysfunction. Furthermore, due to the retrospective nature of the study, a few supplementary patients either missed the echocardiographic follow-up sessions or did not have the echocardiograms at a time interval that matched the criteria of the study proposal. It is to be noted that only one patient was excluded due to excessive shadowing from the prosthetic valve precluding an adequate echocardiographic RV functional assessment.

\section{Conclusion}

The echocardiographic data mentioned details pertaining to the heterogeneous subject group that had undergone PPVI treatment at a single center for the management of RVOT obstruction and/or PR. This patient group was heterogeneous on the basis of their ages, obtained diagnoses, and RVOT morphologies. The study depicted the immediate short-term echocardiographic benefits of PPVI during early-term (1-year) follow-up. As discussed previously, the results obtained were indicative of a significant relief in the RV outflow obstruction and the near-elimination of regurgitation. However, longer-term follow-up studies are required to ascertain the sustained benefits and longevity of this modality of management.

\section{Acknowledgment}

The authors are very thankful to all the associated personnel who contributed to this research.

\section{Conflict of Interest}

Al Asmari Own, Atiyah Merna, Al Essa Mohammed, Almohaizy Omar, Al Sahari Atif, Mohsin Shazia, Al Khalaf Khalaf, and Al Najashi Khalid declare that they have no conflict of interest. This research received no specific grant from any funding agency in the public, commercial, or not-for-profit sectors.

\section{Human Rights Statements and Informed Consent}

All procedures followed were in accordance with the ethical standards and was performed in accordance with the Helsinki Declaration of 1964 and later versions. Informed consent was obtained from all patients for being included in the study.

\section{Bibliography}

1. Biernacka EK., et al. "Percutaneous pulmonary valve implantation - state of the art and Polish experience". Postepy Kardiol Interwencyjnej 13 (2017): 3-9.

2. Conte S., et al. "Homograft valve insertion for pulmonary regurgitation late after valveless repair of right ventricular outflow tract obstruction". European Journal of Cardio-Thoracic Surgery 15 (1999): 143-149.

3. Perron J., et al. "Valved homograft conduit repair of the right heart in early infancy". The Annals of Thoracic Surgery 68 (1999): 542-548.

4. Bando K., et al. "Outcome of pulmonary and aortic homografts for right ventricular outflow tract reconstruction". The Journal of Thoracic and Cardiovascular Surgery 109 (1995): 509-517.

5. Homann M., et al. "Reconstruction of the RVOT with valved biological conduits: 25 years experience with allografts and xenografts". European Journal of Cardio-Thoracic Surgery 17 (2000): 624-630.

6. Dearani JA., et al. "Late follow-up of 1095 patients undergoing operation for complex congenital heart disease utilizing pulmonary ventricle to pulmonary artery conduits". The Annals of Thoracic Surgery 75 (2003): 399-411. 
7. Boethig D., et al. "Evaluation of 188 consecutive homografts implanted in pulmonary position after 20 years". European Journal of Cardio-Thoracic Surgery 32 (2007): 133-142.

8. Stark J., et al. "Fate of subpulmonary homograft conduits: determinants of late homograft failure". The Journal of Thoracic and Cardiovascular Surgery 115 (1998): 506-514.

9. Oosterhof T., et al. "Long-term follow-up of homograft function after pulmonary valve replacement in patients with tetralogy of Fallot". European Heart Journal 27 (2006): 1478-1484.

10. Shebani SO., et al. "Right ventricular outflow tract reconstruction using Contegra valved conduit: natural history and conduit performance under pressure". European Journal of CardioThoracic Surgery 29(2006):397-405.

11. Kanter KR., et al. "One hundred pulmonary valve replacements in children after relief of right ventricular outflow tract obstruction". The Annals of Thoracic Surgery 73 (2002): 18011806.

12. Bouzas B., et al. "Pulmonary regurgitation: not a benign lesion". European Heart Journal 26 (2005): 433-439.

13. Karamlou T., et al. "Surgery insight: late complications following repair of tetralogy of Fallot and related surgical strategies for management". Nature Clinical Practice Cardiovascular Medicine 3 (2006): 611-622.

14. Cesnjevar R., et al. "Late pulmonary valve replacement after correction of Fallot's tetralogy". Thoracic and Cardiovascular Surgery 52 (2004): 23-28.

15. Bielefeld MR., et al. "Reoperative homograft right ventricular outflow tract reconstruction". The Annals of Thoracic Surgery 71 (2001): 482-487.

16. Bonhoeffer P., et al. "Percutaneous replacement of pulmonary valve in a right-ventricle to pulmonary-artery prosthetic conduit with valve dysfunction". Lancet 356 (2000): 1403-1405.

17. Lurz P., et al. "Percutaneous pulmonary valve implantation: impact of evolving technology and learning curve on clinical outcome". Circulation 117 (2008): 1964-1972.

\section{Assets from publication with us}

- Prompt Acknowledgement after receiving the article

- Thorough Double blinded peer review

- Rapid Publication

- Issue of Publication Certificate

- High visibility of your Published work

Website: www.actascientific.com/

Submit Article: www.actascientific.com/submission.php

Email us: editor@actascientific.com

Contact us: +919182824667

Citation: Al Asmari Own., et al. "Echocardiographic Assessment at 1-Year Follow-Up Post-Percutaneous Pulmonary Valve Implantation at the Prince Sultan Cardiac Center: A Single-Center Experience". Acta Scientific Medical Sciences 4.8 (2020): 46-50. 